\title{
Efecto del endoparásito Prosorhynchoides sp. (Trematoda: Bucephalidae) en la capacidad de nado sostenido del baunco Girella laevifrons (Osteichthyes: Kyphosidae)
}

Effect of the endoparasite Prosorhynchoides sp. (Trematoda: Bucephalidae) on the sustained swimming capacity of the sea chub Girella laevifrons (Osteichthyes: Kyphosidae)

\author{
Melissa Rebolledo' ${ }^{1}$, Mauricio F. Landaeta ${ }^{2}$ y Gabriela Muñoz ${ }^{1}$
}

\begin{abstract}
${ }^{1}$ Laboratorio de Parasitología Marina, Escuela de Biología Marina, Facultad de Ciencias del Mar y de Recursos Naturales, Universidad de Valparaíso, Av. Borgoño 16344, Viña del Mar, Chile. gabriela.munoz@cienciasdelmar.cl

${ }^{2}$ Laboratorio de Ictioplancton (LABITI), Escuela de Biología Marina, Facultad de Ciencias del Mar y de Recursos Naturales, Universidad de Valparaíso, Av. Borgoño 16344, Viña del Mar, Chile
\end{abstract}

\begin{abstract}
This study analyzed whether the trematode Prosorhynchoides sp., at metacercaria stage, affects the sustained swimming capacity of the intertidal juvenile sea chub Girella laevifrons. Fifteen non-parasitized and 15 experimentally infested fish were used in this study. Wild fish were infested under laboratory conditions by cercaria from an infected mussel Perumytilus purpuratus. The parasite mainly infested the tail muscles of the fish. The maximum time of sustained swimming of each fish was measured through a swimming camera. Parasitized fish showed less time of sustained swimming and traveled less distance estimated than non-parasitized fish. This result was independent of fish body size, condition factor and trematode abundance. Therefore, Prosorhynchoides sp. affects the swimming capacity of juvenile G. laevifrons that might influence the migration and distribution of the fish.
\end{abstract}

Key words: Sustained swimming, juvenile fish, sea chub, parasite abundance

\section{INTRODUCCIÓN}

La velocidad y resistencia de nado son características fundamentales para la supervivencia de los animales acuáticos, ya que les otorga la capacidad de nado adecuada para alimentarse, reproducirse, escapar y esconderse de depredadores (Videler \& Wardle 1991). Además, determina el desplazamiento de una especie, factor importante en la definición de los límites de hábitat con respecto a otros organismos (Fulton 2010).

Basándose en el tiempo y los procesos bioquímicos de suministro energético de un organismo, el nado de los peces se ha clasificado como: explosivo, prolongado y sostenido (Beamish 1978). El nado explosivo es súbito e intenso, y permite conocer la máxima velocidad alcanzada por el pez (Beamish 1978). La musculatura utiliza la glucólisis anaeróbica para generar energía, proceso que acumula ácido láctico y produce fatiga por la acidificación de tejidos (Hill et al. 2006), consecuentemente, este movimiento no es mantenido por largo tiempo y suele durar menos de 15 s (Beamish 1978). Por otro lado, el nado prolongado utiliza la máxima actividad aeróbica en un pez nadando a velocidades en incremento y usualmente termina en fatiga antes de $200 \mathrm{~min}$ (Hammer 1995, Plaut 2001). El nado sostenido, por su parte, se mantiene a velocidad constante empleando catabolismo aeróbico para producir la energía necesaria para abastecer al organismo durante el ejercicio (Hill et al. 2006), por lo cual no hay acidificación de los tejidos y puede llevarse por periodos prolongados, superiores a $200 \mathrm{~min}$ (Beamish 1978).

La capacidad de nado depende de diversos factores, tales como características propias del pez: morfología de las aletas, edad (Fulton \& Bellwood 2002), tamaño (Videler $\&$ Wardle 1991, Fulton \& Bellwood 2002) y forma corporal del pez (Hammer 1995), así como de factores externos como la temperatura del agua (Videler \& Wardle 1991, Green \& Fisher 2004, Zeng et al. 2009), agentes tóxicos dañinos (Hammer 1995), y la presencia de parásitos (Coleman 1993, Barber et al. 2000, Binning et al. 2012).

Los parásitos podrían afectar la locomoción y el comportamiento de su hospedero, ya sea como un efecto secundario de la infección o como una manipulación adaptativa con el propósito de facilitar su trasmisión (Mouritsen \& Poulin 2002). Los parásitos además pueden ejercer un impacto en la capacidad del hospedero para responder a los estímulos externos, modificando los 
patrones normales de comportamiento (Binning et al. 2012) mediante la alteración de la eficiencia energética sobre la respiración, la circulación, la locomoción o la resistencia física (Barber 2007), lo que consecuentemente afectaría algún aspecto de su movimiento. Por ejemplo, Ascocotyle pachycystis infecta al pez Cyprinodon variegatus obstruyendo su flujo sanguíneo, lo que conlleva a disminuir significativamente el tiempo de nado antes de fatigarse (Coleman 1993).

El tremátodo endoparásito Prosorhynchoides sp. se encuentran naturalmente en estado de esporoquisto en el manto del bivalvo intermareal Perumytilus purpuratus, de donde salen larvas cercarias, las que nadan libremente hasta infestar la musculatura de bauncos juveniles Girella laevifrons (Tschudi, 1846) (Muñoz et al. 2014). El parásito se enquista en la musculatura de este pez quedando en estado de metacercaria.

Poco se sabe de los efectos que tienen los tremátodos en estado de metacercaria en sus hospederos. Las metacercarias que se alojan en la musculatura de un hospedero pueden comprometer las fibras musculares, afectar el flujo sanguíneo capilar o las terminaciones nerviosas. Cualquiera de estas posibilidades, puede tener una influencia importante en la capacidad de nado de hospederos acuáticos. Por lo tanto, se espera que el parásito Prosorhynchoides sp. disminuya el tiempo de nado sostenido de bauncos juveniles, lo que además puede estar directamente relacionado con la abundancia de parásitos. De este modo, el presente estudio evaluó el efecto del endoparásito Prosorhynchoides sp. en la capacidad de nado sostenido de juveniles de G. laevifrons.

\section{MATERIALES Y MÉTODOS}

\section{RECOLECCIÓN DE PECES}

Se recolectaron 54 ejemplares de bauncos juveniles de $G$. laevifrons desde pozas intermareales rocosas de Montemar, Chile central (32॰57'22,34's, 71³3'01,13'W), entre enero y abril 2013, en periodos de baja marea. Para la recolección de peces se utilizó el anestésico AQUI-S ${ }^{\circledR}$, previamente diluido en relación 1:10 con agua potable, que fue adicionada en pequeñas cantidades en las pozas que fueron muestreadas. Con ayuda de redes de manos, los peces fueron capturados y puestos en cubetas con agua de mar libres de anestésico para su recuperación. Se seleccionaron peces juveniles de longitud total (LT) entre 1,5 y $3,5 \mathrm{~cm}$ debido a que la cámara de natación tenía un diámetro de 3,6 cm diámetro x 7,5 cm de largo.
En el laboratorio, los peces se aclimataron en acuarios de $22 \mathrm{~L}$ de capacidad con agua de mar fresca, la cual era remplazada día por medio. Para que la temperatura fuera relativamente constante se utilizó un calefactor sumergible el cual mantuvo la temperatura en $20^{\circ} \mathrm{C}$. Para mantener la oxigenación del agua se utilizaron bombas de aire. Los peces fueron alimentados con frondas frescas de Ulva lactuca una vez al día y puestos en ayuno durante $48 \mathrm{~h}$ antes de ser sometidos a la prueba de nado.

\section{DETERMINACIÓN DE LA CAPACIDAD DE NADO}

El tiempo total de nado fue determinado en una cámara de natación cerrada con recirculación de agua. El sistema estuvo compuesto por 2 bombas sumergibles, con regulación del caudal, que impulsaban el agua por cañerías de PVC de $4 \mathrm{~cm}$ de diámetro. La cámara de natación consistió en un tubo de acrílico transparente $(3,6 \mathrm{~cm}$ de diámetro x 7,5 cm de largo), en posición horizontal, con una tapa en la parte superior por donde el pez era introducido. En los extremos de la cámara se ubicaron mallas de $1 \mathrm{~mm}$ de abertura, que evitaban la salida del pez del área de observación. A los costados de la cámara se ubicaron llaves de paso para regular el caudal y cerrar el flujo del agua. En la salida del sistema, el último tramo del tubo por donde el agua salía, se ubicó un contador de agua para consumo residencial de $0,03 \mathrm{~m}^{3} \mathrm{~h}^{-1} \mathrm{de}$ sensibilidad que permitió cuantificar el caudal de agua que pasó durante el tiempo de duración del experimento. Complementariamente, se implementó un flujómetro ultrasónico Simtech PTTN-100, de mayor precisión $( \pm 1 \%$ del valor medido), para ajustar los valores de caudales de agua en cada medición del nado de los peces. Sin embargo, debido a que el movimiento del pez afectaba la lectura del flujómetro ultrasónico, las correcciones de caudal fueron realizadas sin peces. Para evitar la desoxigenación del agua, se incluyó una bomba de aire al sistema, en el recipiente que recibía al agua de descarga. También se utilizó un calefactor de agua que permitió mantener la temperatura estable en $20^{\circ} \mathrm{C} \pm 1^{\circ} \mathrm{C}$. El sistema de natación completo estuvo dentro de una recámara $(1,1 \mathrm{~m}$ de alto $\mathrm{x}$ 1,4 m de largo x 0,4 m de fondo) cubierta con láminas plásticas opacas que evitaban la perturbación de los peces durante las mediciones de nado.

La medición de nado sostenido fue realizada para cada pez en forma individual. Cada ejemplar fue introducido en la cámara de natación, donde permaneció nadando por 10 min a una velocidad moderada de $6,38 \pm 0,08 \mathrm{~cm} \mathrm{~s}^{-1}$; luego de este tiempo de acondicionamiento, la velocidad fue incrementada a $23,21 \pm 0,003 \mathrm{~cm} \mathrm{~s}^{-1}$, que fue la velocidad 
máxima del experimento. Los peces que no nadaron contra la corriente de forma adecuada, es decir, aquellos que se apoyaron a las paredes de la cámara o las mallas, o presentaron nado errático fueron retirados del sistema y no fueron utilizados nuevamente. Cuando los peces nadaron adecuadamente, el punto de detención del experimento ocurrió cuando estos no lograron seguir nadando contra la corriente afirmándose a la malla ubicada a la salida de la cámara de natación. El tiempo total de nado sostenido por pez fue registrado en minutos. También se calculó la distancia recorrida para cada pez, estimada como la multiplicación entre la velocidad de nado y el tiempo total de nado sostenido.

Una vez terminada la medición, los peces experimentados fueron puestos en contenedores individuales con oxigenación, alimento y temperatura estable para su recuperación durante $48 \mathrm{~h}$. Posteriormente, los peces fueron sacrificados con una sobredosis de anestésico, para la disección y recolección de parásitos. Se buscó ecto y endoparásitos, revisando distintos órganos y partes del cuerpo incluyendo la musculatura. Los parásitos recolectados fueron identificados y contabilizados para cada pez. Aquellos peces que no tenían parásitos fueron denominados como el grupo PNP (= peces no parasitados). Todos los peces disectados fueron medidos y pesados. Con estos datos, a cada individuo se le calculó el índice de condición de Fulton, mediante la fórmula $\mathrm{K}=$ peso/ longitud ${ }^{3}$.

\section{INFESTACIÓN DE PARÁSITOS EN LABORATORIO}

Para la infestación de peces, se utilizó el tremátodo Prosorhynchoides sp. obtenidos del bivalvo mitílido Perumytilus purpuratus naturalmente infestado con este parásito (Muñoz et al. 2004). Aproximadamente, 200 individuos del bivalvo fueron recolectados desde Montemar (Chile central) y puestos individualmente en contenedores plásticos de $30 \mathrm{~mL}$ con agua de mar y revisados diariamente bajo microscopio estereoscópico, para registrar cuáles de ellos se encontraban infectados, mediante la detección de las larvas cercarias libres en el agua. Los bivalvos parasitados fueron separados y utilizados para la infestación de peces.

La infestación de peces se realizó de forma directa, dejando un ejemplar de bivalvo parasitado dentro del acuario durante 48 a $72 \mathrm{~h}$. Los peces estuvieron en ayuna durante las $48 \mathrm{~h}$ previo a la determinación de nado. El éxito de la infestación fue determinando por la presencia de metacercarias en la aleta caudal del pez observado bajo un microscopio estereoscópico luego de la medición de nado (Figs. 1A, B). Para determinar que el parásito correspondiera a Prosorhynchoides sp., se analizó su morfología; cuerpo ovalado de 0,30-0,35 mm de longitud, la larva contenida en la metacercaria presenta un único ciego redondeado y grande, y una vesícula excretora larga y obscura. Estas características fueron fácilmente observables sin necesidad de abrir los quistes (Muñoz et al. 2014).

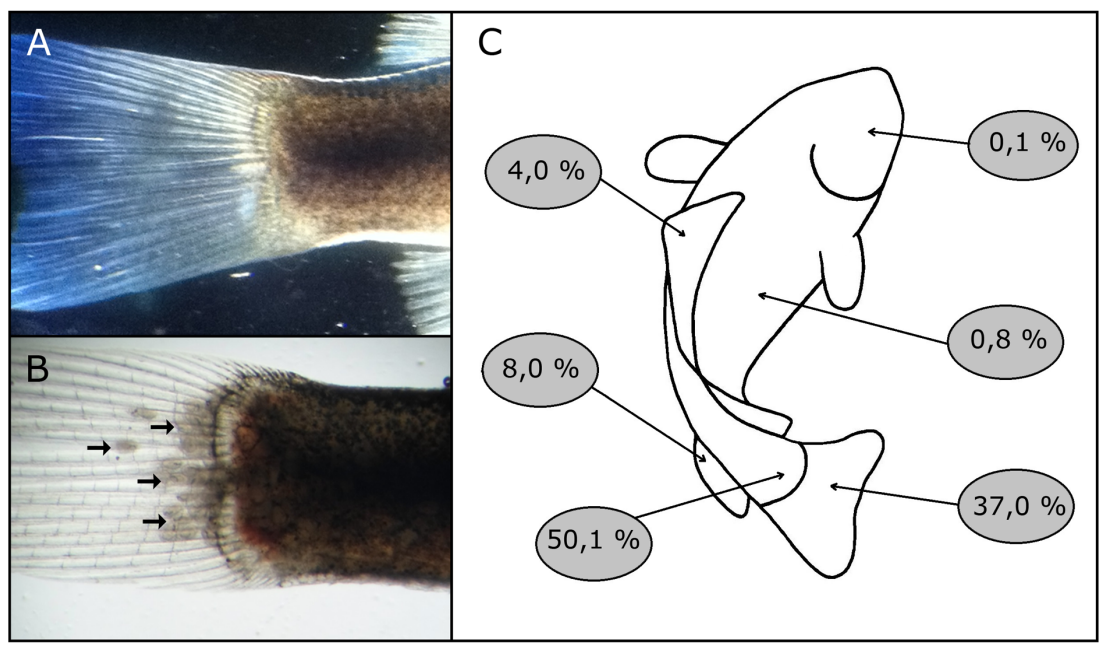

Figura 1. A-B. Fotografías de la aleta caudal de Girella laevifrons obtenidas en un microscopio estereoscópico (aumento 6x). A) Pez no parasitado, B) pez parasitado con metacercarias de Prosorhynchoides sp. (indicadas por flechas), y C) representación gráfica de los sitios de infección de las metacercarias con su respectivo porcentaje de prevalencia / A-B. Photographs of the Girella laevifrons caudal fin, obtained in a stereomicroscope (6X of magnification). A) Non-parasitized, B) parasitized fish with Prosorhynchoides sp. metacercariae (indicated by arrows), and C) display of the infection sites of metacercariae with its percentage of prevalence 
Los peces parasitados (determinados como PP) fueron luego analizados en la capacidad de nado sostenido y examinados para determinar si tenían algún tipo de ecto o endoparásito. Particularmente, las metacercarias presentes en el pez fueron registradas en su sitio de infección, observadas en sus características morfológicas y contabilizadas. Complementariamente, para establecer indirectamente que el grupo de bauncos recolectados del mismo lugar no se encontraban parasitados naturalmente antes del experimento, se analizó un grupo de peces $(\mathrm{n}=$ 24) de similares longitudes corporales, provenientes del mismo sector y periodo de muestreo. De este análisis, se encontró que los peces no presentaban ningún tipo de parásito.

Los grupos de PNP y PP fueron comparados para detectar diferencias significativas en su tamaño corporal (LT y peso), índice de Fulton, tiempo de nado y distancia recorrida, mediante pruebas de $t$-Student, previa confirmación de normalidad de los datos mediante la prueba de Kolmogorov-Smirnov (Zar 1996). La relación entre los tiempos de nado sostenido respecto a la LT del pez entre los grupos de PNP y PP, fueron comparados en sus pendientes e interceptos, por medio del análisis de covarianza de una vía (ANCOVA, Zar 1996). También se realizaron correlaciones de Pearson entre la abundancia de parásitos, el índice de condición de Fulton y el tiempo de nado de PP y PNP. Todos los análisis se realizaron con el paquete estadístico PAST 3.01 (Hammer et al. 2001). El nivel de significación utilizado fue de $5 \%(P<0,05)$ para todos los análisis estadísticos.

\section{RESULTADOS Y DISCUSIÓN}

De los 54 peces recolectados se trabajó con 15 peces pertenecientes al grupo PNP y 15 pertenecientes al grupo PP. El resto de los peces fueron descartados del experimento de nado debido a 2 factores que podrían arrojar errores en las mediciones: lesiones en la aleta caudal $(n=4)$ y nado errático al comienzo del experimento $(\mathrm{n}=4$ PNP y 3 PP). Adicionalmente, algunos peces murieron en cautiverio $(n=3)$, mientras que otros fueron liberados cuando sobrepasaron 7 días en cautiverio sin experimentación $(n=10)$.

No se detectaron diferencias significativas en la LT, peso e índice de Fulton entre ambos grupos (Tabla 1). Sin embargo, el tiempo de nado y la distancia recorrida de los PP fue significativamente menor que en los PNP (Tabla 1). Se encontró una relación positiva entre el tiempo de nado sostenido y la LT de los peces; en los PNP esta relación fue estadísticamente significativa $(\mathrm{r}=0,56, P=$ $0,03)$, pero en los PP no lo fue $(\mathrm{r}=0,28, P=0,31)$. De estas relaciones lineales, se encontró que la pendiente fue similar entre ambos grupos de peces $\left(\mathrm{F}_{(1,27)}=1,22, P<\right.$ 0,277), sin embargo, las medias ajustadas del tiempo de nado fueron significativamente distintas (ANCOVA: $\left.\mathrm{F}_{(1,27)}=18,92, P<0,001\right)$, en donde los PP tuvieron menor promedio de tiempo de nado que los PNP(Fig. 2). Además, el tiempo de nado sostenido de peces no se correlacionó con el índice de Fulton de PP $(\mathrm{r}=0,22, P=0,42)$ ni de PNP (r=0,06, $P=0,83)$.

En los PP se encontraron sólo metacercarias de Prosorhynchoides sp., y ningún otra especie de parásito. Se recolectó un total de 927 metacercarias, de las cuales $50,1 \%$ se localizaron en la musculatura del pedúnculo caudal, siendo el mayor porcentaje en comparación a otros 5 sitios de infección (Fig. 1C). En el grupo de PP, no se encontraron correlaciones significativas entre la abundancia de parásitos y tiempo de nado $(\mathrm{r}=0,27, P=$ $0,94)$, ni en la abundancia de parásitos y el índice de Fulton $(\mathrm{r}=0,57, P=0,06)$.

Tabla 1. Promedio \pm desviación estándar de las variables biológicas medidas en juveniles de baunco Girella laevifrons / Mean values \pm standard deviation of biological variables measured in juvenile fish of Girella laevifrons

\begin{tabular}{lcccc}
\hline \multirow{2}{*}{ Variable } & \multirow{2}{*}{$\begin{array}{c}\text { No Parasitados } \\
(\mathrm{n}=15)\end{array}$} & $\begin{array}{c}\text { Parasitados } \\
(\mathrm{n}=15)\end{array}$ & \multicolumn{2}{c}{ T-student } \\
\cline { 4 - 5 } & $2,37 \pm 0,51$ & $2,31 \pm 0,24$ & 0,364 & 0,718 \\
LT $(\mathrm{cm})$ & $0,15 \pm 0,09$ & $0,12 \pm 0,05$ & 0,905 & 0,372 \\
Peso $(\mathrm{g})$ & $0,0096 \pm 0,0021$ & $0,0094 \pm 0,0024$ & 0,207 & 0,836 \\
Índice de Fulton & $342,9 \pm 124,9$ & $156,5 \pm 121,5$ & 4,140 & 0,002 \\
Tiempo nadado (min) & & & & \\
Distancia recorrida $(\mathrm{m})$ & $4.978,4 \pm 1.814,1$ & $2.278,4 \pm 1.767,6$ & 4,128 & 0,002 \\
\hline
\end{tabular}


Figura 2. Regresión lineal entre tiempo de nado sostenido y longitud total (LT) de peces no parasitados (PNP) y peces parasitados (PP) de Girella laevifrons con metacercarias del trematodo Prosorhynchoides sp. / Lineal regression between sustained swimming time and total length (LT) of non-parasitized (PNP) and parasitized (PP) fish of Girella laevifrons with metacercaria of the trematode Prosorhynchoides sp.

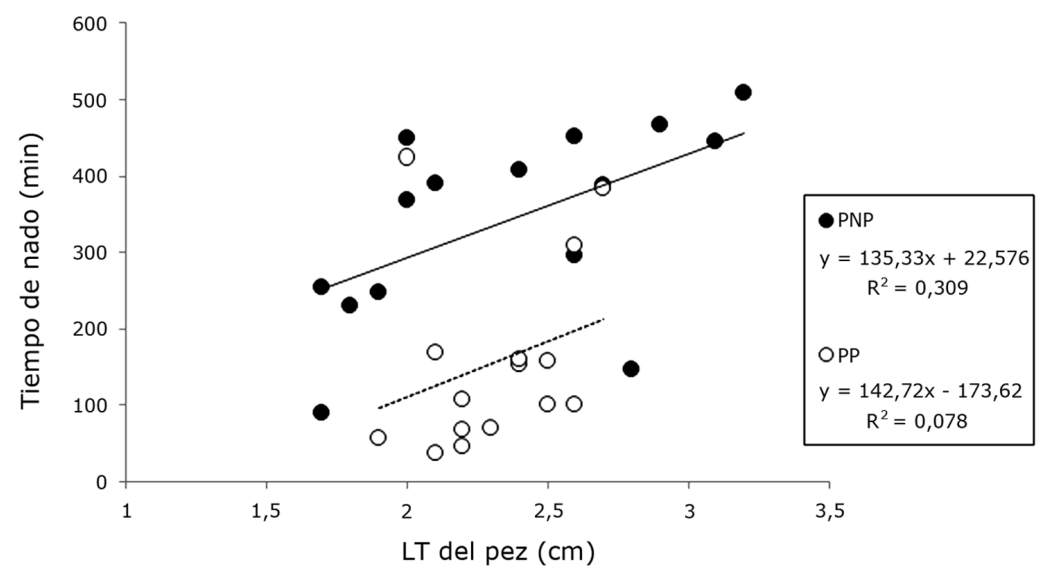

Los resultados de estos experimentos sugieren que los parásitos tienen un efecto negativo en la capacidad de nado de G. laevifrons, debido a que el tiempo de nado sostenido fue significativamente menor en PP que en PNP. G. laevifrons es capaz de resistir distintos estresores, tales como factores ambientales, antrópicos y/o ecológicos (Carrasco-Malio et al. 2014, Pulgar et al. 2014), por lo tanto la manipulación adicional en los PP, al momento de infectarlos con cercarias, podría tener poco efecto en la capacidad de nado, y no invalidaría la comparación entre ambos grupos de peces (PP y PNP). Además, el tiempo de nado no se correlacionó con la abundancia de parásitos; peces que tenían pocas metacercarias (abundancia mínima encontrada=9) mostraron similar tiempo de nado que aquellos que tenían muchas metacercarias (más de 200). Es decir, la condición de estar parasitado, sin importar la abundancia de parásitos en el pez, fue el factor que influyó en el tiempo y distancia de nado en juveniles de G. laevifrons.

Poulin (1994) indicaron que los efectos de la abundancia de parásitos en el comportamiento del hospedero podrían ser acumulativos, por lo tanto, a medida que aumenta la intensidad parasitaria, el grado de cambios de comportamiento debiera aumentar aproximadamente de forma lineal, mientras que la infección podría aumentar el coste metabólico de la locomoción (Barber et al. 2000). Sin embargo, en el presente estudio se encontró que pocas metacercarias ejercen un efecto negativo, posiblemente a nivel muscular o metabólico, pero que no se ve aumentado con la presencia de muchas metacercarias, es decir que la disminución del tiempo de nado es independiente a la abundancia de éstos parásitos. Esto podría explicarse porque durante el enquistamiento del tremátodo, el hospedero responde con una fuerte reacción en sus tejidos, lo que puede incluir inflamación, hemorragias localizadas y destrucción celular, efectos que son metabólicamente exigentes para el pez (Esch \& Fernández 1993), lo cual puede ser gatillado por pocos individuos parásitos. Además, considerando que cerca del $50 \%$ de las metacercarias estaban en la base de aleta caudal (en los huesos hipurales) donde hay poca musculatura, por lo tanto unas pocas metacercarias producirían un efecto significativo en estos tejidos, afectando el movimiento de la aleta caudal y por ende el nado de los peces.

Algunos antecedentes previos establecen que la longitud corporal es factor determinante en el tiempo de nado sostenido de peces (Videler \& Wardle 1991, Fulton \& Bellwood 2002), mientras que el parasitismo afecta la condición del pez (Esch \& Fernández 1993). Esch \& Fernández (1993) demostraron que existe una relación negativa y significativa entre número tremátodos de la especie Uvulifer ambloplitis enquistados naturalmente y el coeficiente de condición Fulton en el pez de agua dulce Lepomis macrochirus. Sin embargo, esto no fue observado en el presente estudio debido a que el tiempo de post-infección con metacercarias de Proshorynchoides sp. en G. laevifrons fue de 5 días. Es decir, a una pequeña escala temporal los peces no experimentaron algún efecto significativo en el peso o la longitud corporal; de esta forma, el factor de condición fue similar entre PP y PNP.

Las abundancias de Prosorhynchoides sp.en $G$. laevifrons variaron entre 9 y 245 metacercarias por pez. Algunos presentaron más de lo que se había reportado en infecciones naturales (hasta 40 metacercarias, Muñoz et al. 2014). A pesar de esta diferencia, se encontró que la capacidad de nado de G. laevifrons no se correlacionó 
con la abundancia de metacercarias, por lo tanto, la capacidad de nado de bauncos parasitados naturalmente debiera mostrar un comportamiento similar a lo observado en este estudio. Esto tiene una importante connotación ecológica, ya que la reducción en la capacidad de nado facilita la depredación, así como también disminuye el potencial de advección de la especie, lo que puede repercutir en la distribución y supervivencia del hospedero, especialmente en la zona intermareal, que es una zona altamente variable causada por el oleaje, las mareas, el viento y la temperatura (Gibson 1999).

\section{Agradecimientos}

Este estudio fue financiado por el proyecto FONDECYT Regular 1120868. Se agradecen los comentarios y sugerencias de 3 evaluadores anónimos que ayudaron a mejorar una versión preliminar del manuscrito.

\section{LITERATURA CITADA}

Barber I. 2007. Parasites, behavior and welfare in fish. Applied Animal Behavior Science 104: 251-264.

Barber I, D Hoare \& J Krause. 2000. Effects of parasites on fish behavior: a review and evolutionary perspective. Reviews in Fish Biology and Fisheries 10:131-165.

Beamish FWH. 1978. Swimming capacity. In: Hoar WS \& JD Randall (eds). Fish physiology, pp. 101-187. Academic Press, New York.

Binning S, D Roche \& C Layton. 2012. Ectoparasites increase swimming cost in a coral reef fish. Biology Letters 9(1): 20120927<doi: 10.1098/rsbl.2012.0927>

Carrasco-Malio A, M Díaz, M Mella, MJ Montoya, A Miranda, MF Landaeta, G Sánchez \& ME Hidalgo. 2014. Are the intertidal fish highly resistant to UV-B radiation? A study based on oxidative stress in Girella laevifrons (Kyphosidae). Ecotoxicology and Environmental Safety 100: 93-98.

Coleman F. 1993. Morphological and physiological consequences of parasites encysted in the bulbus arteriosus of an estuarine fish, the sheepshead minnow, Cyprinodon variegatus. The Journal of Parasitology 79(2): 247-254.

Esch G \& J Fernández. 1993. A functional biology of parasitism. Ecological and evolutionary implications, 337 pp. Champan \& Hall, London.

Fulton C. 2010. The role of swimming in reef fish ecology. In: Domenici P \& B Kapoor (eds). Fish swimming: an ethoecological perspective, pp. 374-406. Science Publishers, Enfield.
Fulton C \& D Bellwood. 2002. Ontogenetic habitat use in labrid fishes: an ecomorphological perspective. Marine Ecology Progress Series 236: 236-262.

Gibson RN. 1999. Movement and homing in intertidal fishes. In: Horn MH, LM Martin \& MA Chotkowski (eds). Intertidal fishes: Life in two worlds, pp. 97-125. Academic Press, London.

Green B \& R Fisher. 2004. Temperature influences swimming speed, growth and larval duration in coral reef fish larvae. Journal of Experimental Marine Biology and Ecology. 299: 115-132.

Hammer C. 1995. Fatigue and exercise test with fish. Comparative Biochemistry and Physiology A112(1): 120.

Hammer $\emptyset$, DAT Harper \& PR Ryan. 2001. PAST: Paleontological statistics software package for education and data analysis. Palaeontologia Electronica 4(1): 1-9.

Hill G, A Wyse \& W Richard.2006. Fisiología animal, 1038 pp. Editorial Médica Panamericana, Madrid.

Mouritsen KN \& R Poulin. 2002. Parasitism, community structure and biodiversity in intertidal ecosystems. Parasitology 124: 101-117.

Muñoz G, I Valdivia \& Z López. 2014. Life cycle of Prosorhynchoides carvajali (Trematoda: Bucephalidae) in the intertidal zone of central Chile. Journal of Helminthology FirstView: 1-9. <10.1017/S0022149X1400054>

Plaut I. 2001. Critical swimming speed: its ecological relevance. Comparative Biochemistry and Physiology A 131: 41-50.

Poulin R. 1994. Evolution of parasite manipulation of host behaviour. A theoretical analysis. Parasitology 109: S109S118.

Pulgar J, P Lagos, D Maturana, M Valdés, M Aldana \& VM Pulgar. 2014. Effect of UV radiation on habitat selection by Girella laevifrons and Graus nigra. Journal of Fish Biology (en prensa).<doi: 10.1111/jfb.12566>

Videler J \& C Wardle. 1991. Fish swimming stride by stride: speed limits and endurance. Reviews in Fish Biology and Fisheries 1: 23-40.

Zar JH. 1996. Biostatistical analysis, 718 pp. Prentice-Hall International, Englewood Cliffs.

Zeng L, Z Cao, S Fu, J Peng \& Y Wang. 2009. Effect of temperature on swimming performance in juvenile southern catfish (Silurus meriodinalis). Comparative Biochemistry and Physiology A 153: 125-130. 